\title{
Development of a Photoresist for Etching Designs in Glass
}

\author{
Chester I. Pope and Raymond Davis
}

\begin{abstract}
The preparation of a light-sensitive coating from phenol-formaldehyde resin is described. The light-sensitive coating may be used as a resist or mask in etching scales or reticule patterns in glass. A new etching solution was developed for use with this resist, and it is also recommended for fine line etching with wax resists.
\end{abstract}

\section{Introduction}

Etched glass scales and reticules are commonly produced by using a wax resist as a mask. A thin layer of wax coated on the glass is engraved or ruled in such a manner that the design or pattern is accurately positioned and the glass is uncovered in the engraved areas. The design is etched into the glass with hydrofluoric acid. After removing the resist, the etched areas are usually filled with an opaque pigment to increase the contrast and legibility. The workers in this field have a need for a light-sensitive coating, or resist, so that duplicate etchings may be made by contact printing, as in photography.

The problem of finding a suitable substance for making a light-sensitive resist is difficult because the glass must be etched with hydrofluoric acid, which attacks all of the known light-sensitive coatings. The coatings invariably crack, undercut, decompose, or become permeable to the etching solution before the etching is complete. Procedures using an undercoat, such as silver or a varnish, under the light-sensitive resist did not prove satisfactory.

Phenol-formaldehyde resin was known to be quite resistant to the action of hydrofluoric acid, and it seemed possible that a thin layer coated on glass might serve as a resist for hydrofluoric-acid etching after development of the image. Doelker [1] ${ }^{1}$ patented a process in which a phenol-formaldehyde resin, sensitized with ammonium dichromate, was recommended as an etching resist for zinc plates. Beebe, Murray, and Herlinger [2] patented the use of light-sensitive phenol-formaldehyde resin for photographic processes. They sensitized the resin with iodine, iodoform, etc., and, after exposure, developed the coating in a solution of about equal parts of alcohol and water. The resin made according to their directions was quite soft, remained tacky too long after coating for contact printing, and was low in light sensitivity. In the course of the present investigation it was found that the softness and tackiness of the resin was due in part to the presence of methyl alcohol in the formaldehyde solution used in the synthesis of the resin. The addition of methyl alcohol to trioxymethylene and phenol, in a concentration corresponding to that generally found in 40-

\footnotetext{
1 Figures in brackets indicate the literature references at the end of this paper.
}

percent formaldehyde solutions, yielded a soft, tacky resin. Therefore, trioxymethylene was used instead of the 40-percent aqueous solution of formaldehyde for the production of the phenol-formaldehyde resin in this work. Also the alcohol-water developer used by Beebe, Murray, and Herlinger [2] was not a satisfactory developer for the resin on glass. A resorcinolformaldehyde resin was also made and was found useful as an additive in the phenol-formaldehyde resin.

\section{Synthesis of the Phenol-formaldehyde Resin and the Resorcinol-formaldehyde Resin}

\subsection{Synthesis of the Phenol-formaldehyde (State-A) Resin}

Baekeland $[3,4,5]$ divided the phenol-formaldehyde reaction during resinification into three stages, $\mathrm{A}, \mathrm{B}$, and $\mathrm{C}$, which are characterized by the appearance and solubility of the resin. The A-stage, or initial product, is a low molecular weight condensation product soluble in acetone; the B-stage, or intermediate condensation product, is swelled by acetone but not soluble in it; and the C-stage, or final condensation product, is infusible and completely insoluble in acetone. The resin used for the photoresist is that formed in the A-stage and is condensed to the state where it is just soluble in 95-percent ethyl alcohol. The resin is a thermosetting phenolic, which slowly passes into the B-stage on drying at room temperature.

Sodium acetate, a weak alkaline salt, proved to be the best catalyst for the condensation of the phenol and formaldehyde. It yielded a resin that had the greatest light sensitivity and which was readily developable by an organic solution. Other salts tested as catalysts were potassium acetate, lithium acetate, calcium acetate, barium acetate, and sodium formate. These salts produced resins that were low in light sensitivity and could not be as easily developed as the resin formed with sodium acetate. Acids such as hydrochloric acid act as strong catalysts and produce a resin that is not as light sensitive as that produced by the weak alkaline catalyst, sodium acetate, and the condensation 
reaction is difficult to control because the resin passes rapidly from the A-stage to the B-stage.

Investigation of the condensation reaction showed that the ratio of 1 mole of phenol, 3 moles of trioxymethylene, 6 moles of water, and 0.3 mole of sodium acetate $\left(\mathrm{CH}_{3} \mathrm{COONa} .3 \mathrm{H}_{2} \mathrm{O}\right)$, produced a satisfactory light-sensitive resin that was not too soft. Decreasing the sodium acetate gave a softer resin, which is less light sensitive.

The following formula and procedure were worked out for synthesizing the light-sensitive resin:

Phenol

Water

Sodium acetate $\left(\mathrm{CH}_{3} \mathrm{COONa} 3 \mathrm{H}_{2} \mathrm{O}\right)$

$282 \mathrm{~g}$

$324 \mathrm{ml}$

$122 \mathrm{~g}$

Trioxymethylene (paraformaldehyde)

$270 \mathrm{~g}$

The phenol and trioxymethylene were USP grade, and the sodium acetate was reagent quality, meeting ACS specifications. Distilled water was used in the synthesis and tap water in washing the resin.

The apparatus consisted of a 3,000-ml roundbottomed flask fitted with a reflux condenser by means of a ground-glass joint and mounted on a ring stand. The apparatus should be located in a chemical hood. The chemicals were added to the flask in the order listed in the formula followed by the addition of boiling ships made from an unglazed porcelain plate. The mixture was heated through an asbestos pad with a Bunsen burner to a gentle boil. The ring stand was rocked gently and frequently to prevent superheating until the boiling began. The boiling was continued throughout the period of the reaction, and the time required was $2 \frac{1}{2}$ to $3 \frac{1}{2} \mathrm{hr}$. The temperature of the boiling solution during the reaction and before precipitation was $105^{\circ} \mathrm{C}$. Toward the end of the condensation reaction the solution became cloudy. It became opaque to transmitted light about $20 \mathrm{~min}$ before two definite phases developed, accompanied by foaming. After the two phases appeared, a gentle and frequent rocking of the ring stand was helpful in preventing the resin, which slowly precipitates from the boiling mixture, from sticking to the flask. After the two phases appeared, the boiling was continued about $5 \mathrm{~min}$, and the hot reaction mixture was immediately poured into cool water while being rapidly stirred, thus completing the precipitation of the resin from the hot reaction mixture. The resin was washed and kneaded in about five changes of water and tested for degree of condensation. This test was made by taking about $10 \mathrm{~g}$ of the resin batch, heating it on the steam bath in a small porcelain dish while stirring with a glass rod, and noting the time for the resin to pass into the B-stage. This time was observed visually when the resin became rubbery, stringy, or lumpy and was the point at which all or most of the resin became insoluble in 95-percent ethyl alcohol. If the resin has condensed close enough to the B-stage, the small sample of resin should become insoluble in 95-percent ethyl alcohol after being heated for $10 \mathrm{~min}$ on the steam bath. If the time was much greater than $10 \mathrm{~min}$, the precipitated resin batch was heated on the steam bath in a large porcelain dish and stirred constantly with a glass rod, and the time of the heating was estimated from the time required for the small sample to become insoluble in 95-percent ethyl alcohol. With experience, the operator will find no difficulty in determining the end-point of the condensation. As a safeguard against carrying the condensation of the precipitated resin too far on the steam bath, the resin batch may be cooled with water at intervals and a small sample tested for degree of condensation as described above. The resin passes rapidly from $\mathrm{A}$-stage to $\mathrm{B}$-stage at a temperature of $100^{\circ} \mathrm{C}$, and if the resin shows any signs of stringiness, or a tendency to be rubbery and lumpy, it should be cooled immediately by stirring in cool water. The resin is usable even if a few insoluble particles appear in the alcohol solution, but it should be discarded if essentially insoluble in alcohol. The highest degree of light sensitivity and developability were obtained by condensing the resin as close to the B-stage as possible, without the resin becoming insoluble in 95-percent ethyl alcohol.

After the resin had been condensed to the desired state and cooled, as much of the water as possible was pressed out and the resin dissolved immediately in $700 \mathrm{ml}$ of methyl ethyl ketone (2-butanone) of a good commercial grade, free from acid or any substance that would react with iodoform. Then 500 $\mathrm{g}$ of anhydrous sodium sulfate was added and the resin solution allowed to stand for several days, with occasional shaking. After filtering the resin solution it was ready for sensitizing and use. The amount of resin, determined from the water-free solutions of different batches, gave yields of 220 to $300 \mathrm{~g}$. The resin solutions contained from 22.5 to $30 \mathrm{~g}$ of resin per $100 \mathrm{ml}$ of solution. The concentration of the resin in the filtered solution was determined by noting the volume, evaporating $10 \mathrm{ml}$ of the solution to dryness in a tared 50-ml beaker, and weighing.

For use as a light-sensitive resist coating, the concentration of the resin was adjusted to $20 \mathrm{~g}$ of resin per $100 \mathrm{ml}$ of solution and sensitized by adding $1 \mathrm{~g}$ of iodoform for each $10 \mathrm{~g}$ of resin. The iodoform used was Eastman Kodak Cat. No. P-341. Just before use, the sensitized resin solution was filtered through a coarse fritted-disk filtering funnel without pressure. Sensitized resin solutions have been usable after storage for 2 years in a refrigerator. Unsensitized resin solutions stored in the dark at room temperature were still usable after 3 years. The phenol-formaldehyde resin required about 2 to 3 times more exposure to actinic light than the wellknown cold top enamel to render the coating insoluble in the developer solution.

A second formula containing ethylene glycol as part of the solvent was developed for the synthesis of the resin. The resin made by this last formula was generally sufficiently condensed when precipitated so that no further treatment was necessary on the steam bath. However, it should be tested for degree of condensation, as described above, before dissolving it in the methyl ethyl ketone. The resin has the same sensitivity as that made by the first formula but contains a trace of ethylene glycol. 
Ethylene glycol, because of its hydroscopic nature, caused a slight decrease in the light sensitivity of the resin coating at high relative humidities. The effect was nil at a relative humidity of 50 percent or less.

The following alternative formula was worked out for synthesizing the phenol-formaldehyde resin:

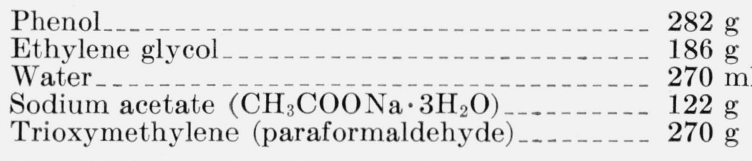

The ethylene glycol was of reagent grade. The apparatus and procedure for producing the resin were the same as described for the previous formula. The time required for the reaction was 2 to $3 \mathrm{hr}$. The temperature of the boiling solution during the reaction and before precipitation was $107.5^{\circ} \mathrm{C}$. The reaction mixture remained clear until very close to the end of the reaction. The solution became cloudy, and the boiling was continued about $3 \mathrm{~min}$ after it had become opaque to transmitted light. The resin was immediately precipitated from the hot reaction mixture by pouring into cool water with rapid stirring. The resin was treated in the same manner as that obtained from the first formula.

\subsection{Synthesis of the Resorcinol-formaldehyde Resin}

The resorcinol-formaldehyde resin, because of its reactivity and chemical similarity to the phenolformaldehyde resin, should improve the sensitivity and resist properties of the phenol-formaldehyde resin, and this was found to be true. A resin, soluble in 95-percent ethyl alcohol, was produced from the condensation of resorcinol and formaldehyde. Although this resin was light sensitive, it was not possible to develop the image satisfactorily. It is somewhat more resistant to hydrofluoric acid than the phenol-formaldehyde resin. The addition of 5percent of the resin, based on the weight of the phenol-formaldehyde resin, increased the sensitivity about 25 percent, increased the intensity of the dyeing of the image during development, and improved its resist properties slightly.

The following formula and procedure were used in synthesizing the alcohol-soluble resorcinol-formaldehyde resin:

$\begin{array}{ll}\text { Resorcinol } & 55 \mathrm{~g} \\ \text { Water } & \text { Sodium sulfate, anhydrous } \\ \text { Formaldehyde solution, } 40 \% \ldots & 12.5 \mathrm{~g} \\ \end{array}$

The resorcinol was Eastman Kodak Cat. No. $\mathrm{P}-222$, and the sodium sulfate was reagent quality, meeting ACS specifications. The formaldehyde was USP grade. Distilled water was used both in the synthesis and in washing the precipitate. The chemicals were added to a $250-\mathrm{ml}$ Erlenmeyer flask and brought into solution. The reaction mixture was stored without agitation in a constant-temperature room at $22 \pm 1^{\circ} \mathrm{C}$. The solution was clear after $24 \mathrm{hr}$, but after $40 \mathrm{hr}$ a precipitate began to settle out. At the end of $140 \mathrm{hr}$ the reaction mixture was diluted to $1,500 \mathrm{ml}$, and then $100 \mathrm{~g}$ of anhydrous sodium sulfate was added. The sodium sulfate salts out the resin during the reaction and also on dilution before filtering. The precipitated resin was removed with suction by means of a Buchner filtering funnel. The precipitate was thoroughly washed, air-dried overnight, and finally dried in the dark in a desiccator over anhydrous calcium chloride. When the reaction time was extended much beyond $140 \mathrm{hr}$ the resin became insoluble in 95-percent ethyl alcohol.

The rate of the condensation reaction increased with increase in temperature, accompanied by an increase in the yield of an alcohol-insoluble resin. At $100^{\circ} \mathrm{C}$ the reaction was very rapid, and the resin was completely insoluble in ethyl alcohol. It was difficult to obtain a good yield of the alcoholsoluble resorcinol-formaldehyde resin when the reaction was carried out at a temperature much above $25^{\circ} \mathrm{C}$

The dried resin, when stored in a dry brown bottle in the dark at room temperature, was still soluble in 95-percent ethyl alcohol after 2 years. The resin, when dissolved in methyl ethyl ketone over anhydrous sodium sulfate at a concentration of about 20 $\mathrm{g}$ per $100 \mathrm{ml}$ of solution, was still usable after 3 years of storage at room temperature.

\section{Development of the Exposed Resin Coating}

After suitable exposure in contact with a master positive, the unexposed areas of the resin coating were dissolved out (developed), and the exposed areas were dyed simultaneously by means of an organic solution developed for use on this resin coating. The developer solution was made up as follows:

$\begin{array}{ll}\text { Ethyl alcohol, } 95 \% & 1,000 \mathrm{ml} \\ \text { Normal butyl alcohol, bp } 117 \text { to } 117.8^{\circ} \mathrm{C}-- & 1,000 \mathrm{ml} \\ \text { Methyl ethyl ketone, bp } 79 \text { to } 80.5^{\circ} \mathrm{C}_{-}- & 200 \mathrm{ml} \\ \text { Methyl violet_- } & 2 \mathrm{~g} \\ \text { Basic fuchsin (aniline red, magenta) } \ldots \ldots \ldots- & 2 \mathrm{~g}\end{array}$

Before use the solution was filtered through a coarse frittered-disk filtering funnel.

The ethyl alcohol, normal butyl alcohol, and methyl ethyl ketone were of reagent grade and were used without distillation or drying. The developer solution contained about 3 percent of water. The mixture of methyl violet and basic fuchsin dyed the resin coating a heavy blue-purple and was found to be the best combination of dyes tested. Methyl violet dyed the resin coating blue-green; basic fuchsin, red; and malachite green, green. Another developer solution was also made up without the dye for use in removing the excess dye that collected on the surface during the developing procedure. The exposed resin coating was developed by immersing the coated specimen in the developer-dye solution for 30 to $40 \mathrm{sec}$ with gentle agitation and rinsing it 2 or 3 times in developer solutions that contained no dye. Although the development took place in 30 to $40 \mathrm{sec}$ at room temperature, there were no adverse effects on the resin coating if the developing time was extended for as long as $2 \mathrm{~min}$. 


\section{Etching Solution}

After developing and drying, the resistance of the resin coating to hydrofluoric acid was increased by baking it in an oven between 100 to $135^{\circ} \mathrm{C}$ for 1 to $3 \mathrm{hr}$. Concentrated hydrofluoric acid $(48 \%)$ attacked the phenol-formaldehyde resin coating. The problem was to find a solvent for the hydrofluoric acid that would delay its attack on the resin coating and prevent the formation of insoluble salts during the etching. Experimentally the answer was found in using hydrofluoric acid in aqueous solution with phosphoric acid. Also, phosphoric acid assisted the hydrofluoric acid in etching the glass [6]. The phosphoric acid retarded the attack of the hydrofluoric acid on the developed resin and gave a clear etch when sufficient water was added. The following formula was developed:

Ortho phosphoric acid, $85 \% \ldots 100 \mathrm{ml}$ Water Hydrofluoric acid, $48 \% \ldots$

The phosphoric and hydrofluoric acids were of reagent quality, meeting $\mathrm{ACS}$ specifications. The etching solution was easy to make up as no noticeable heating took place when the chemicals were mixed. The etching solution may be used as soon as it is made up, and, if stored in a wax pot fitted with a tight cover, it will keep for several months. If the developed phenol-formaldehyde resin coating is not too thin (not less than 0.00004 in. thick), and has been baked, it will withstand the etching solution for periods up to $15 \mathrm{sec}$ at room temperature. A 5 -sec etch is recommended for fine line work where the etch is to be filled chemically. A deep etch may be obtained by etching several times for 3 to 4 sec, washing and drying at $100^{\circ} \mathrm{C}$ for about $30 \mathrm{~min}$ between each etch. At the end of the etch, the glass specimen was immersed immediately in a 20 -percent solution of sodium hydroxide solution to neutralize the hydrofluoric acid and to remove the resin coating.

\section{Conclusion}

Many organic substances are sensitive to or hardened by actinic light, but it is generally a difficult problem to find a developer solution that will dissolve out the unexposed areas of the resin and not attack the exposed areas. The developer solution dissolved the unexposed light-sensitive resin quickly and yet did not attack the exposed resin, even if the development was extended several times beyond the normal developing time. Iodoform was found to be a very good sensitizer. The addition of 5 percent of the resorcinol-formaldehyde resin, based on the weight of the phenol-formaldehyde resin, improved the sensitivity and resist properties of the phenol-formaldehyde resin, although its presence was not necessary to make the phenolformaldehyde resin function as a resist for etching glass.

The light-sensitive resist made with the phenolformaldehyde resin will not withstand the action of concentrated hydrofluoric acid (48\%). However, the etching solution containing hydrofluoric acid and phosphoric acid may be used for etching times up to 15 sec. An etching time of 5 sec was used to produce fine lines (10 microns wide). Even though the light-sensitive resist will not withstand a long etching time, deep lines may be obtained with the hydrofluoric acid-phosphoric acid etching solution by etching the glass several times for 3 or 4 sec, washing, and drying between each etch.

For the details on the use of the phenol-formaldehyde light-sensitive resist, see National Bureau of Standards Circular 565 [7] Techniques for Ruling and Etching Precise Scales in Glass and Their Reproduction by Photoetching with a New Lightsensitive Resist.

\section{References}

[1] E. Doelker, British Patent 183,817 (1923).

[2] M. C. Beebe, A. Murray, and H. V. Herlinger, U. S. Patent $1,587,270$ (1926).

[3] L. H. Baekeland, Ind. Eng. Chem. 1, 149 (1909).

[4] R. L. Wakeman, The chemistry of commercial plastics, p. 115 (Reinhold Publishing Corp., New York, N. Y., 1947).

[5] C. Ellis, The chemistry of synthetic resins, vol. 1, pp. 295, 342 (Reinhold Publishing Corp., New York, N. Y., 1935).

[6] B. Schweig, Principles and methods of etching and embossing, Glass, 143 (April 1938).

[7] Raymond Davis and Chester I. Pope, NBS Circular 565 (in press).

Washington, April 5, 1955. 